\title{
Optical properties of GaN/AlN multiple quantum wells
}

\author{
T.Y. Lin ${ }^{\mathrm{a}, *}$, Y.M. Sheu ${ }^{\mathrm{b}}$, Y.F. Chen ${ }^{\mathrm{b}}$, J.Y. Lin ${ }^{\mathrm{c}}$, H.X. Jiang ${ }^{\mathrm{c}}$ \\ ${ }^{a}$ Institute of Optoelectronic Sciences, National Taiwan Ocean University, Keelung 202, Taiwan, ROC \\ ${ }^{\mathrm{b}}$ Department of Physics, National Taiwan University, Taipei 106, Taiwan, ROC \\ ${ }^{\mathrm{c}}$ Department of Physics, Kansas State University, Manhattan, KS 6505-2601, USA
}

Received 12 May 2004; accepted 25 May 2004 by P. Wachter

Available online 11 June 2004

\begin{abstract}
Optical properties of GaN/AlN multiple quantum wells (MQW) have been investigated by Raman scattering, photoluminescence and photoluminescence excitation measurements. A careful examination of the Raman spectrum reveals the fact that the constituent layers of GaN/AlN MQWs are well strained. The experimental results of emission and absorption in MQWs were compared with the calculated solutions of the finite quantum well and the bound states involved in the optical transitions were identified. It is found that the interband transitions up to $n=3$ bound state can be observed in the strained GaN/AlN MQWs sample. The temperature dependence of the heavy-hole transitions shows an interesting phenomenon, in which the peak energy first increases with increasing temperature and then decreases with the temperature rapidly. The observation can be explained in a consistent way by the strain effects of lattice mismatch due to the interplay between the thermal expansion of GaN and AlN layers. Our results indicate that pseudomorphic GaN/AlN MQWs with good quality can be readily grown, and their applications in optoelectronics can be expected in the near future.
\end{abstract}

(C) 2004 Elsevier Ltd. All rights reserved.

PACS: 78.66.H; 78.55.E; 78.30.F

Keywords: A. Quantum wells; E. Photoluminescence; E. Strain

III-Nitride semiconductors are of extensive research currently for a wide range of applications, including blue and ultraviolet light-emitting diodes and lasers, as well as high power and high temperature electronics [1-4]. With a large energy gap of $6.2 \mathrm{eV}$, AlN is the least studied materials in III-nitrides [5]. The energy gap as large as $2.8 \mathrm{eV}$ between $\mathrm{GaN}$ and AlN leads to the energy of the interband transitions of GaN/AlN multiple quantum wells (MQWs) higher than that of other heterostructure in III-nitrides. Thus, GaN/AlN MQWs offer many potential applications in optoelectronics, particularly in the ultraviolet regions. However, the study of their optical properties is rather limited. In this letter, we report a detailed study on the optical properties of GaN/AlN MQWs. The results of micro-Raman scattering measurements show that there exists a compressive strain in the $\mathrm{GaN}$

\footnotetext{
* Corresponding author. Tel.: +886-224622192x6703; fax: +886-224634360.

E-mail address: tylin@mail.ntou.edu.tw (T.Y. Lin).
}

well layers and a tensile strain in the AlN barrier layers. Several distinct features were observed in the photoluminescence (PL) and photoluminescence excitation (PLE) spectra. These experimental results were compared with the calculated solutions of the finite quantum well and the bound states involved in the optical transitions were identified. It is found that the interband transitions up to $n=3$ bound state can be observed in the strained GaN/AlN MQWs sample. Moreover, the abnormal blueshifts of the PL peaks with increasing temperature was found and can be explained in a consistent way by the strain effects and the thermal mismatch between $\mathrm{GaN}$ and AlN layers. These results imply that good quality of GaN/AIN MQWs can be readily grown, and their application in optoelectronic devices can be expected in the near future.

The GaN/AlN MQWs studied here was grown by metalorganic chemical-vapor deposition (MOCVD). A $0.5 \mu \mathrm{m}$ AlN epilayer was first deposited on a sapphire substrate with $20 \mathrm{~nm}$ low-temperature AIN buffer layer, followed by five 
periods of GaN/AlN MQWs with well and barrier thickness of 26 and $30 \AA$, respectively. Details of the growth procedures have been reported elsewhere [5]. The Raman spectrum was measured by a Jobin Yvon-Spex T64000 triple-grating micro-Raman spectrometer equipped with a cooled charge-coupled-device array using an $\mathrm{Ar}^{+}$laser with a $514.5 \mathrm{~nm}$ wavelength at room temperature, and the spectra resolution is about $0.5 \mathrm{~cm}^{-1}$. The micro-PL spectra were excited by a $193 \mathrm{~nm}$ ArF-excimer laser with $10 \mathrm{~Hz}$ repetition rate, and 40 ps pulse width recorded by a Jobin Yvon-Spex T64000 single-grating spectrometer. The temperature-dependent micro-PL measurements were accomplished by placing the sample in an open-cycled liquid $\mathrm{He}$ cryostat. The PLE measurement was excited by a Xe-lamp, and the sample was placed in a $15 \mathrm{~K}$ closed-cycle cryostat. The singal was detected by a SPEX $0.85 \mathrm{~m}$ monochromator equipped with a cooled photomultiplier tube (PMT).

The micro-Raman spectrum of the MQW sample recorded in the back-scattering configuration is shown in Fig. 1. Three features are observed at 576, 655, and $748 \mathrm{~cm}^{-1}$, which do not correspond to the phonon frequencies of unstrained wurtzite GaN and AlN [6-11]. According to the selection rules, however, they are quite similar to the phonons of $E_{2}$ and $A_{1}$ (LO) symmetry of the $C_{6 v}$ point group observed in plain hexagonal layers. The relative closeness in frequency with the GaN modes and/or the AlN modes suggests to assign the three features in Fig. 1 to phonons in the corresponding layers of the MQWs. It was found that the GaN $E_{2}$ and $A_{1}(\mathrm{LO})$ phonons shift toward higher frequencies while the AlN $E_{2}$ phonon shifts toward lower frequency. Such shifts may arise from strain, optical phonon confinement, and the zone-folding effect in the superlattice system. If the zone-folding effect dominates the frequency shift, only the $A_{1}$ mode should be shifted, because the zone-folding only influences the phonon modes in which the motion of atoms has the component along the $c$ axis of wurtzite GaN [12]. However, this is not the case as shown in our observation. We therefore can rule out the possibility of

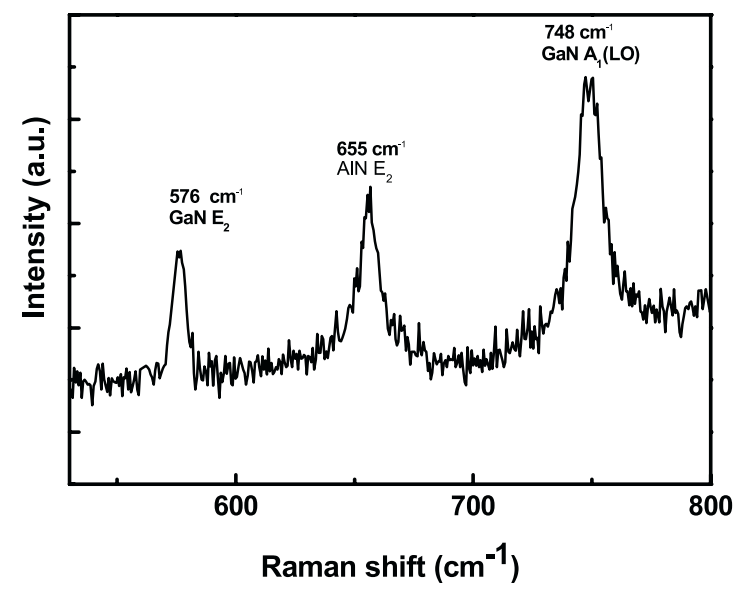

Fig. 1. Room-temperature Raman spectrum of GaN/AlN MQWs. zone-folding effect in spite of that the dispersion curves of the optical phonons of GaN and AlN overlap and there is no gap. On the other hand, optical phonon confinement effect cannot be invoked to explain the blue shift of phonon modes of $\mathrm{GaN}$, due to the overlapping of the phonon density of states of GaN and AlN. Thus, the most plausible explanation of these shifts is the presence of a built-in strain. In the GaN/ AlN MQWs, the lattice constant of bulk GaN is larger than that of bulk AlN. Therefore, the GaN (AlN) layers have compressive (tensile) biaxial strain in the planes parallel to the interfaces to match the lattice constants. Thus, the strains can lead to the blueshifts of the phonon modes in $\mathrm{GaN}$ well and the redshift in AlN barrier as observed in the microRaman spectrum.

Fig. 2 shows the micro-PL spectrum of an GaN/AlN MQWs at low temperature. Two emission peaks at 3.96 and $4.53 \mathrm{eV}$ are observed at $6 \mathrm{~K}$. The peak positions of both these two emission peaks that appear below the bulk AlN emission but above the bulk GaN emission are attributed to confined particle transitions in the quantum wells. To account for the blue shift in the PL spectrum of the GaN/ AlN MQWs, the quantum confined effect and strain effects must be considered. Strain effects due to the lattice mismatch between the GaN well materials and the AlN barriers lead to an increase (decrease) in the band-gap energy of the well (barrier) material providing that the presence of a large concentration of defects in the materials does not prevent from the formation of coherent strain. The changes in values of the band-gap energy due to strain effects were calculated [13-15] and we obtain 3.85, $4.01 \mathrm{eV}$ for $E_{\mathrm{e}-\mathrm{hh}}, E_{\mathrm{e}-l \mathrm{~h}}$ of $\mathrm{GaN}$ and 5.87, $5.74 \mathrm{eV}$ for $E_{\mathrm{e}-\mathrm{hh}}, E_{\mathrm{e}-\mathrm{lh}}$ of AlN. It is found that the observed values for the emission from the GaN quantum wells are both higher than the values calculated. In order to further identify the origin of the peaks, a detailed calculation, including the quantum confined effect and strain effects was performed by solving a one-dimensional finite well Schrödinger equation for electrons and holes in the QWs [16,17]. We obtain the

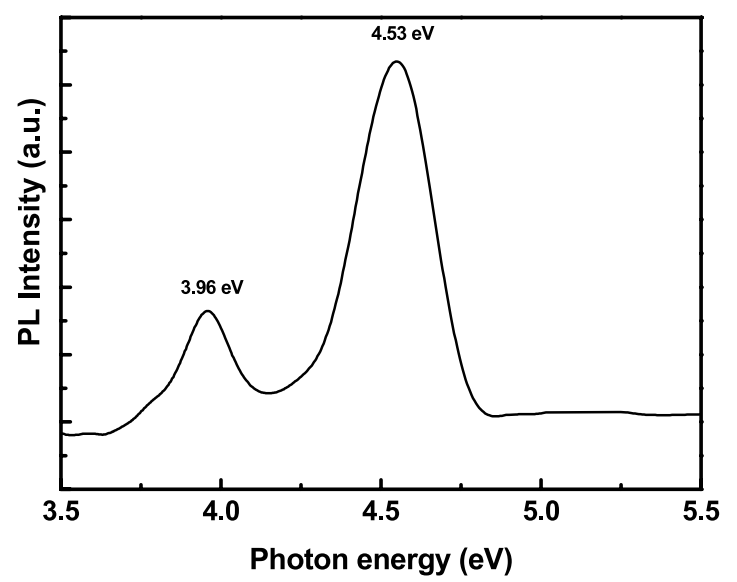

Fig. 2. Photoluminescence spectrum of GaN/AlN MQWS at $6 \mathrm{~K}$. 
allowed transitions, $E_{\mathrm{e} 1-\mathrm{hh} 1}, E_{\mathrm{e} 2-\mathrm{hh} 2}$ and $E_{\mathrm{e} 3-\mathrm{hh} 3}$, which are $4.04,4.55$ and $5.24 \mathrm{eV}$, respectively. In addition, the interband transition, $E_{\mathrm{e} 1-\mathrm{lh} 1}$, is $4.28 \mathrm{eV}$. The calculated results are compared to the measured PL peak energies shown in Fig. 2. The emission lines observed at 3.96 and $4.53 \mathrm{eV}$ correspond to the optical transitions of $E_{\mathrm{e} 1-\mathrm{hh} 1}$, $E_{\mathrm{e} 2-\mathrm{hh} 2}$ in the wells, respectively. Thus, more than one subband exists for both electrons and holes in the wells as expected due to the large band-gap difference between the AlN barrier and the GaN well. Furthermore, the band tilting resulting from the effect of internal electric field [18] makes the electrons and holes closer to the triangular regions in the wells and leads to the discrepancy in the values of the measurement and calculation results. Hence, the measured and the calculated results agree better for the $E_{\mathrm{e} 2-\mathrm{hh} 2}$ transition. Besides, although the low-energy subbands are more populated than the high-energy subbands, their small overlap integral prevents their luminescence from dominating the PL spectrum. The emission intensity of $E_{\mathrm{e} 2-\mathrm{hh} 2}$ transition is therefore higher than that of the $E_{\mathrm{e} 1-\mathrm{hh} 1}$ transition. We point out that all the observations mentioned above can be understood by the strain induced by lattice mismatch between GaN and AlN layers.

Fig. 3 shows the PLE spectrum of GaN/AlN MQWs at low temperature $(15 \mathrm{~K})$. The PLE spectrum is obtained by monitoring the emission of $E_{\mathrm{e} 1-\mathrm{hh} 1}$ transition. The PLE spectrum results from the different optical transitions possible between the quantized electron and hole states. Fig. 3 shows the Gaussian fits for the PLE spectrum and three absorption peaks at $4.25,4.55$, and $5.24 \mathrm{eV}$. By comparing the results with the calculations, the three peaks correspond to the interband transitions, $E_{\mathrm{e} 1-\mathrm{lh} 1}, E_{\mathrm{e} 2-\mathrm{hh} 2}$ and $E_{\mathrm{e} 3-\mathrm{hh} 3}$, respectively. We have therefore demonstrated that the interband absorption up to $n=3$ bound state has been observed in the GaN/AIN MQWs. This PLE result implies

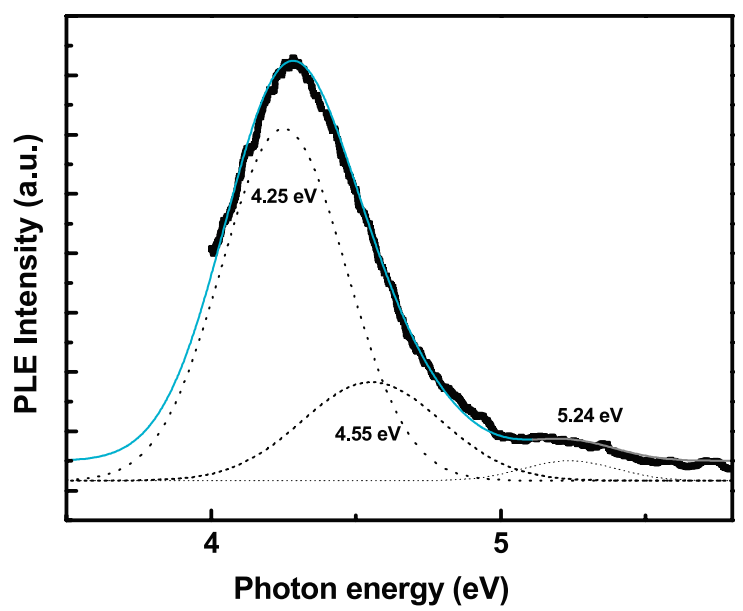

Fig. 3. Photoluminescence excitation spectrum GaN/AlN MQWS at $15 \mathrm{~K}$. The dashed lines show the Gaussian fits for the bound state transitions in MQWs.

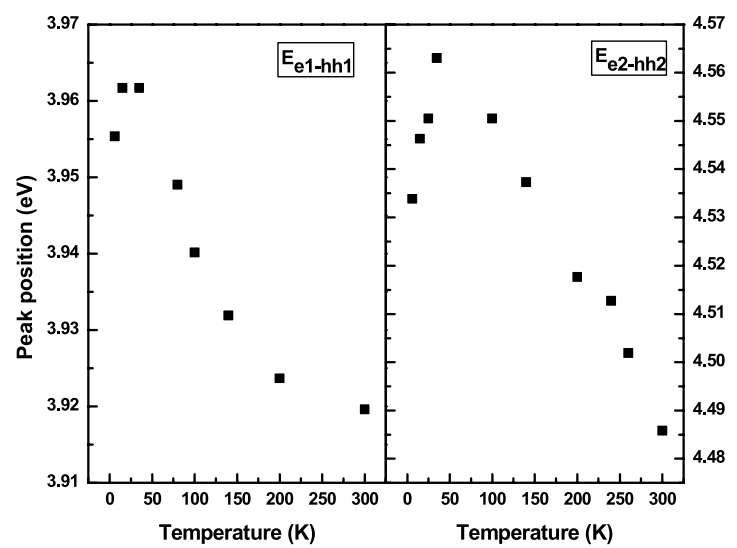

Fig. 4. The luminescence peak positions of $E_{\mathrm{e} 1-\mathrm{hh} 1}$ and $E_{\mathrm{e} 2-\mathrm{hh} 2}$ as a function of the temperature of the AlN/GaN MQWs.

that good quality of GaN/AIN MQW can be readily grown and their applications can be expected in the near future.

Fig. 4 shows the luminescence peak positions of $E_{\mathrm{e} 1-\mathrm{hh} 1}$ and $E_{\mathrm{e} 2-\mathrm{hh} 2}$ as a function of the temperature of the AlN/GaN MQWs sample. The spectrum exhibits an unusual behavior, in which the peak energy first increases with increasing temperature below $40 \mathrm{~K}$ and then decreases with the temperature rapidly. Accordingly, the thermal expansion coefficient of $\mathrm{GaN}$ is $5.59 \times 10^{-6} / \mathrm{deg}$ while that of $\mathrm{AlN}$ is $5.3 \times 10^{-6} / \mathrm{deg}$, which is smaller than that of $\mathrm{GaN}[19]$. As the temperature first increases at low temperature, the $\mathrm{GaN}$ well layers with larger lattice constant and larger thermal expansion coefficient are therefore further compressed. Under biaxial compression, the hydrostatic component of the stress increases the mean band gap, while the axial component splits the degeneracy of the valence band. This causes the increase in transition energy in $\mathrm{GaN}$ wells and leads to the blueshift of peak energy of the PL spectra at the low temperature ranges. As temperature increases further and when the effect of electron-phonon interaction dominates, the transition energy shows the redshift as the usual behavior in which the band gap of a semiconductor decreases with increasing temperature. Thus, the anomalous temperature dependence of the bound state transitions in AlN/GaN MQWs can be explained in a consistent way by the strain effects of lattice mismatch due to the interplay between the thermal expansions of GaN and AlN layers. This result further demonstrates the existence of strain in the pseudomorphic GaN/AlN MQWs.

In conclusion, we have reported a detailed study of optical properties of GaN/AlN MQWs. From the microRaman scattering measurement, we have shown that the $\mathrm{GaN}$ well is under compressive stress, while the AlN barrier is under tensile stress. The experimental results of emission and absorption in MQWs were compared with the calculated solutions by solving a one-dimensional finite well Schrödinger equation and the bound states involved in the optical transitions in QWs were identified. It is found that the 
interband transitions up to $n=3$ bound state can be observed in the strained AlN/GaN MQWs sample. In addition, the temperature dependence of the heavy-hole transitions shows an interesting phenomenon, in which the peak energy first increases with increasing temperature and then decreases with the temperature rapidly. The observation can be explained in a consistent way by the effects of strain induced by the difference between the thermal expansion coefficients of GaN and AlN layers. Our results indicate that pseudomorphic GaN/AlN MQWs with good quality can be readily grown, and their applications in optoelectronics can be expected in the near future.

\section{Acknowledgements}

This work was supported by the Ministry of Education and the National Science Council of the Republic of China.

\section{References}

[1] S. Nakamura, G. Fasol, The Blue Laser Diode: GaN Bassed Light Emitters and Lasers, Springer, Berlin, 1997.

[2] M.A. Khon, Q. Chen, M.S. Shur, B.T. McDermott, J.A Higgins, J. Burm, W.J. Schoff, L.F. Eastman, IEEE Electron Device Lett. 17 (1996) 584.

[3] O. Aktas, Z.F. Fan, A. Botchkarev, S.N. Mohammad, M. Roth, T. Jenkins, L. Kehias, H. Morkoc, IEEE Electron Device Lett. 18 (1977) 293.
[4] Y.F. Wu, B.P. Keller, S. Keller, D. Kapolnek, P. Kozodoy, S.P. Denbaars, U.K. Mishra, Appl. Phys. Lett. 69 (1996) 1438.

[5] K.B. Nam, J. Li, K.H. Kim, J.Y. Lin, H.X. Jiang, Appl. Phys. Lett. 78 (2001) 3690.

[6] G. Wei, J. Zi, K. Zhang, X. Xie, J. Appl. Phys. 82 (1997) 4693.

[7] T. Kozawa, T. Kachi, H. Kano, Y. Taga, M. Hashimoto, J. Appl. Phys. 75 (1994) 1098

[8] A. Tabata, R. Enderlein, J.R. Leite, S.W. da Silvia, J.C. Galzerani, D. Schikora, M. Kloide, K. Lischka, J. Appl. Phys. 79 (1996) 4137.

[9] P. Perlin, A. Polian, T. Suski, Phys. Rev. B 47 (1993) 2874.

[10] K. Karch, J.M. Wagner, F. Bechstedt, Phys. Rev. B 57 (1998) 7043.

[11] A.R. Goñi, H. Siegle, K. Syassen, C. Thomsen, J.-M. Wagner, Phys. Rev. B 64 (2001) 035205

[12] C.H. Chen, Y.F. Chen, A. Shih, S.C. Lee, H.X. Jiang, Appl. Phys. Lett. 78 (2001) 3035.

[13] S.L. Chuang, Physics of Optoelectronic Devices, Wiley, New York, 1995.

[14] S.-H. Park, S.-L. Chuang, J. Appl. Phys. 87 (2000) 353.

[15] I. Vurgaftman, J.R. Meyer, L.R. Ram-Mohan, J. Appl. Phys. 89 (2001) 5815.

[16] M. Suzuki, T. Uenoyama, A. Yanase, Phys. Rev. B 52 (1995) 8132.

[17] K. Kim, W.R.L. Lambrecht, B. Segall, M. van Schilfgaarde, Phys. Rev. B 56 (1997) 7363.

[18] H. Morkoc, Nitride Semiconductors and Devices, Springer, Heidelberg, 1998

[19] S. Yoshida, S. Misawa, S. Gonda, Appl. Phys. Lett. 42 (1983) 427. 